Supporting Information for

\title{
Free-Standing, Ordered Mesoporous Few-Layer Graphene Framework Films Derived from Nanocrystal Superlattices Self-Assembled at the Solid- or Liquid-Air Interface
}

Li Ji, ${ }^{\dagger}$ Guannan Guo, ${ }^{\dagger},{ }^{\dagger}$ Hongyuan Sheng, ${ }^{\dagger}$ Shanli Qin, ${ }^{\dagger}$ Biwei Wang,,${ }^{\dagger}$ Dandan Han,${ }^{\dagger}$ Tongtao $\mathrm{Li}^{\dagger}$ Dong Yang, ${ }^{* *}$ and Angang Dong ${ }^{*}{ }^{\dagger}$

${ }^{\dagger}$ Collaborative Innovation Center of Chemistry for Energy Materials, Shanghai Key Laboratory of Molecular Catalysis and Innovative Materials, and Department of Chemistry, Fudan University, Shanghai 200433, China.

*State Key Laboratory of Molecular Engineering of Polymers and Department of Macromolecular Science, Fudan University, Shanghai 200433, China.

*To whom correspondence should be addressed: yangdong@fudan.edu.cn; agdong@fudan.edu.cn (A.D.) 


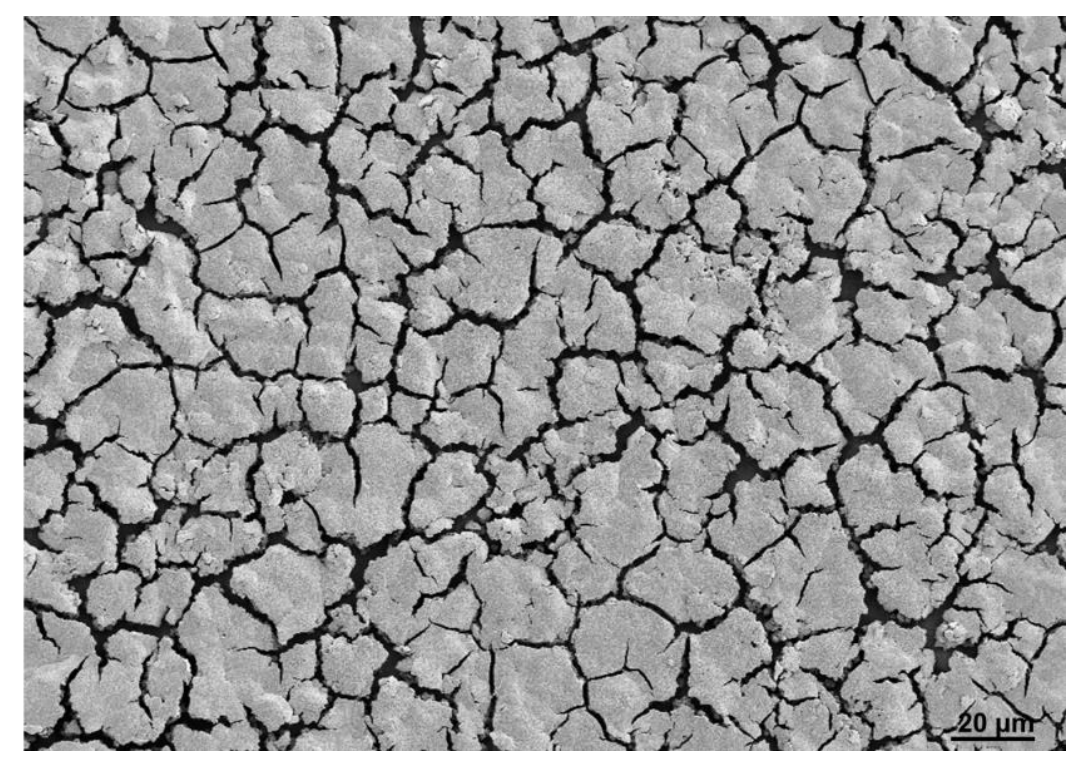

Figure S1. Low-magnification SEM image of discontinuous NC films self-assembled from diluted NC solutions $(\sim 20 \mathrm{mg} / \mathrm{mL})$, showing small and isolated NC domains. 


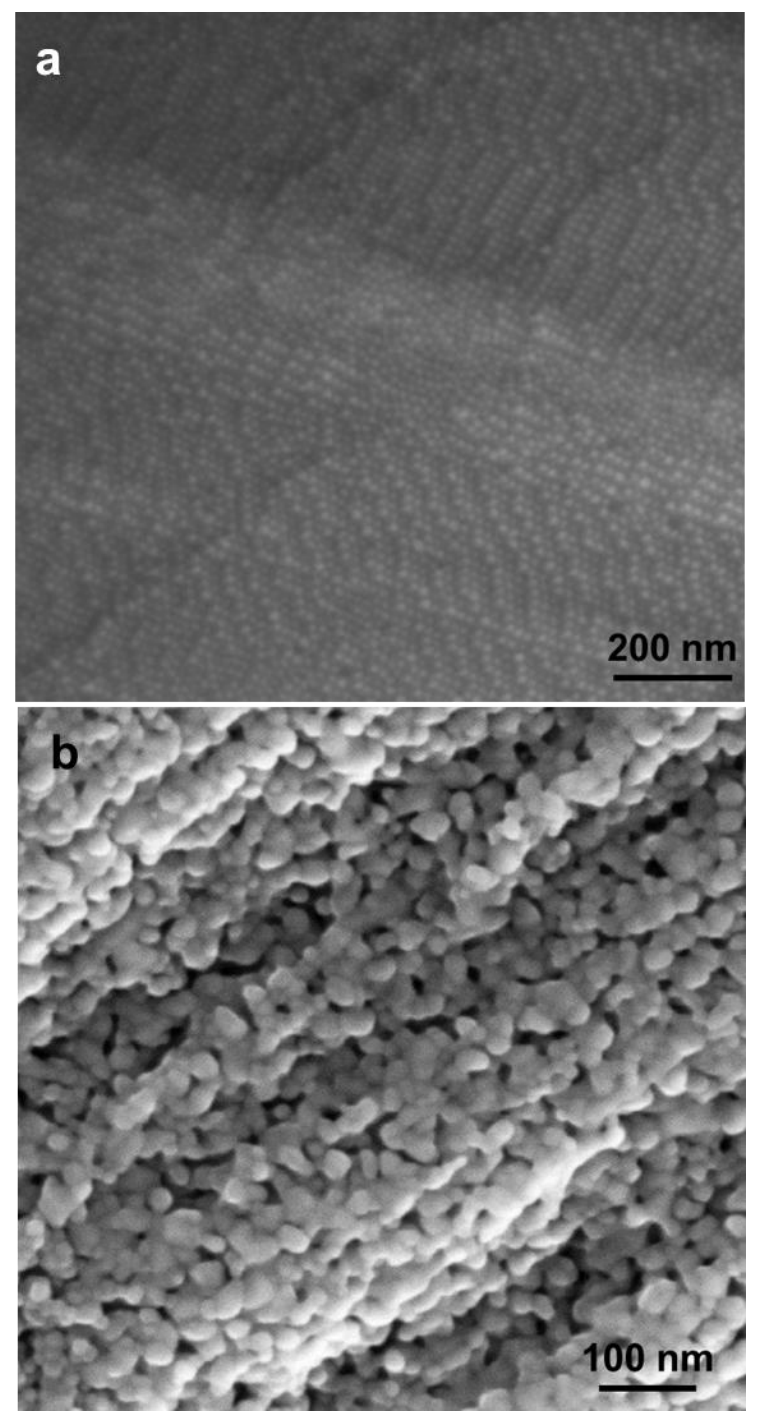

Figure S2. HRSEM images of NC superlattice films (a) before and (b) after heat treatment at $600{ }^{\circ} \mathrm{C}$ under Ar for $2 \mathrm{~h}$, showing the partially sintered NCs after heat treatment. 

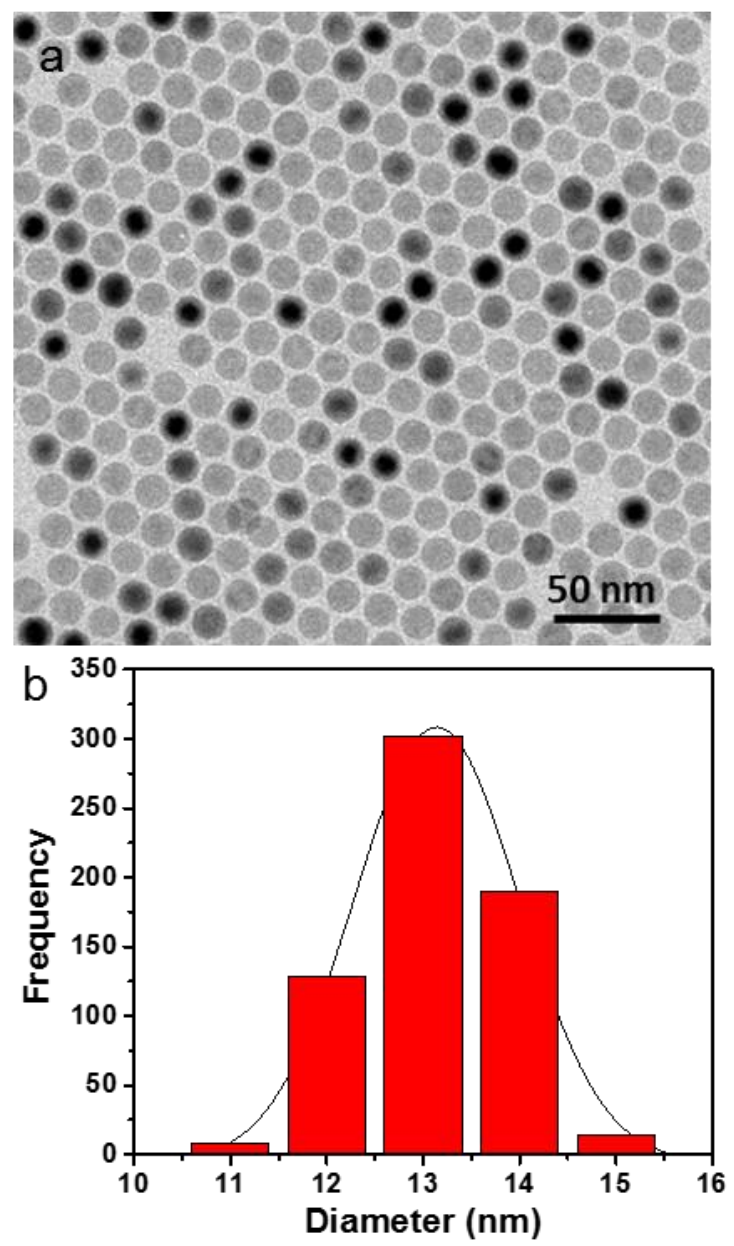

Figure S3. (a) TEM image and (b) particle size distribution histograms of $13 \mathrm{~nm} \mathrm{Fe} 3 \mathrm{O}_{4} \mathrm{NCs}$ used for self-assembly of 2D NC superlattices. The mean diameter of $\mathrm{Fe}_{3} \mathrm{O}_{4} \mathrm{NCs}$ was determined to be $13.1 \mathrm{~nm}$ with a size distribution of $\sim 5 \%$. 


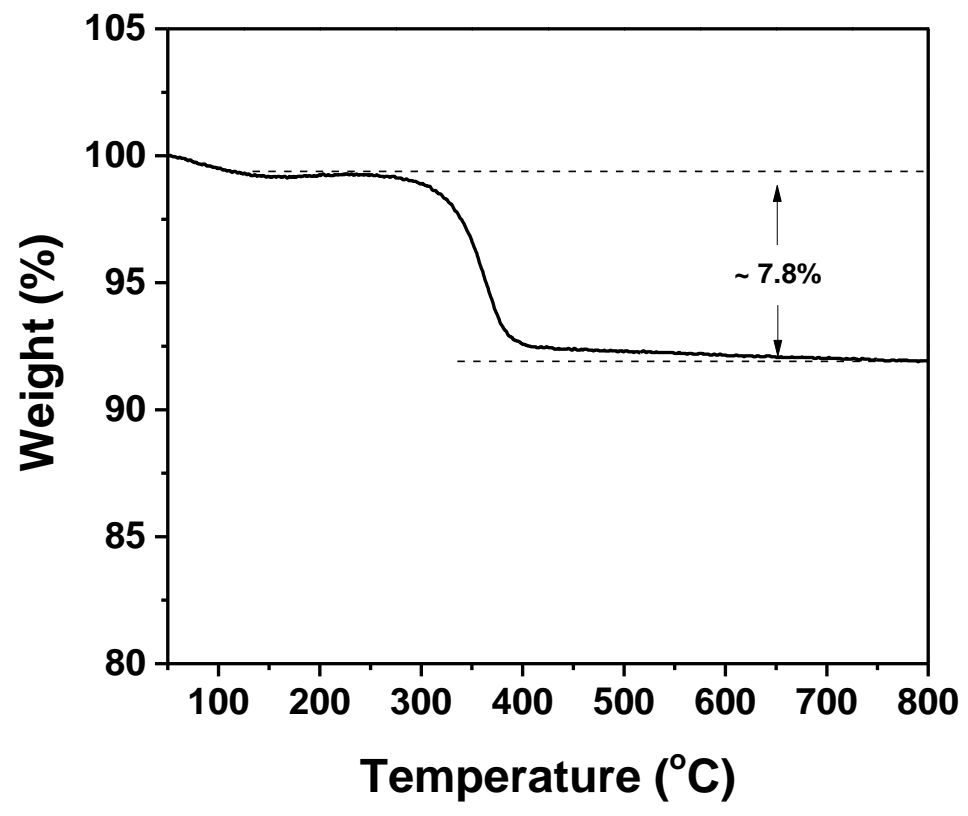

Figure S4. TGA curve of carbonized $\mathrm{Fe}_{3} \mathrm{O}_{4} \mathrm{NC}$ superlattice films measured in air, showing the carbon content was $~ 7.8 \mathrm{wt} \%$. 


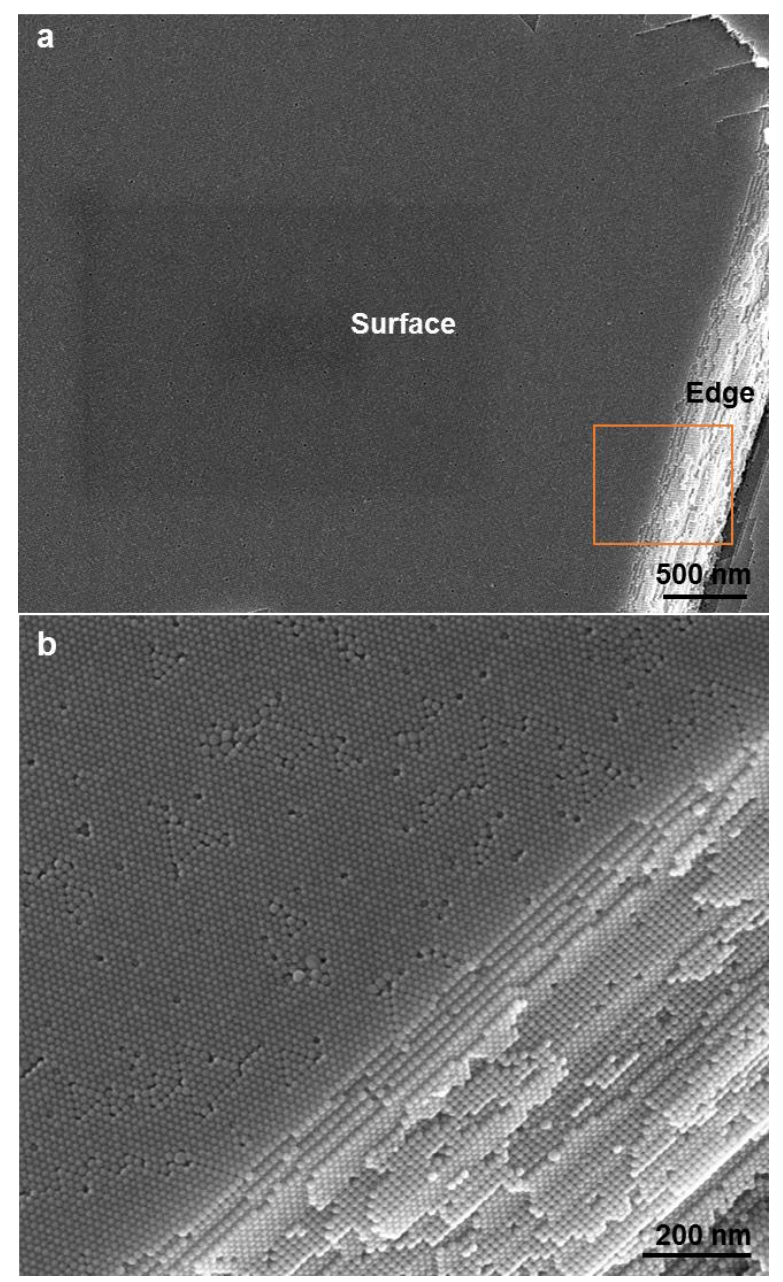

Figure S5. (a) Low-magnification SEM image of the edge of $\mathrm{Fe}_{3} \mathrm{O}_{4} \mathrm{NC}$ superlattice films after ligand carbonization. (b) High-magnification SEM image of the region indicated in (a), showing the high degree of NC ordering across the film thickness. 


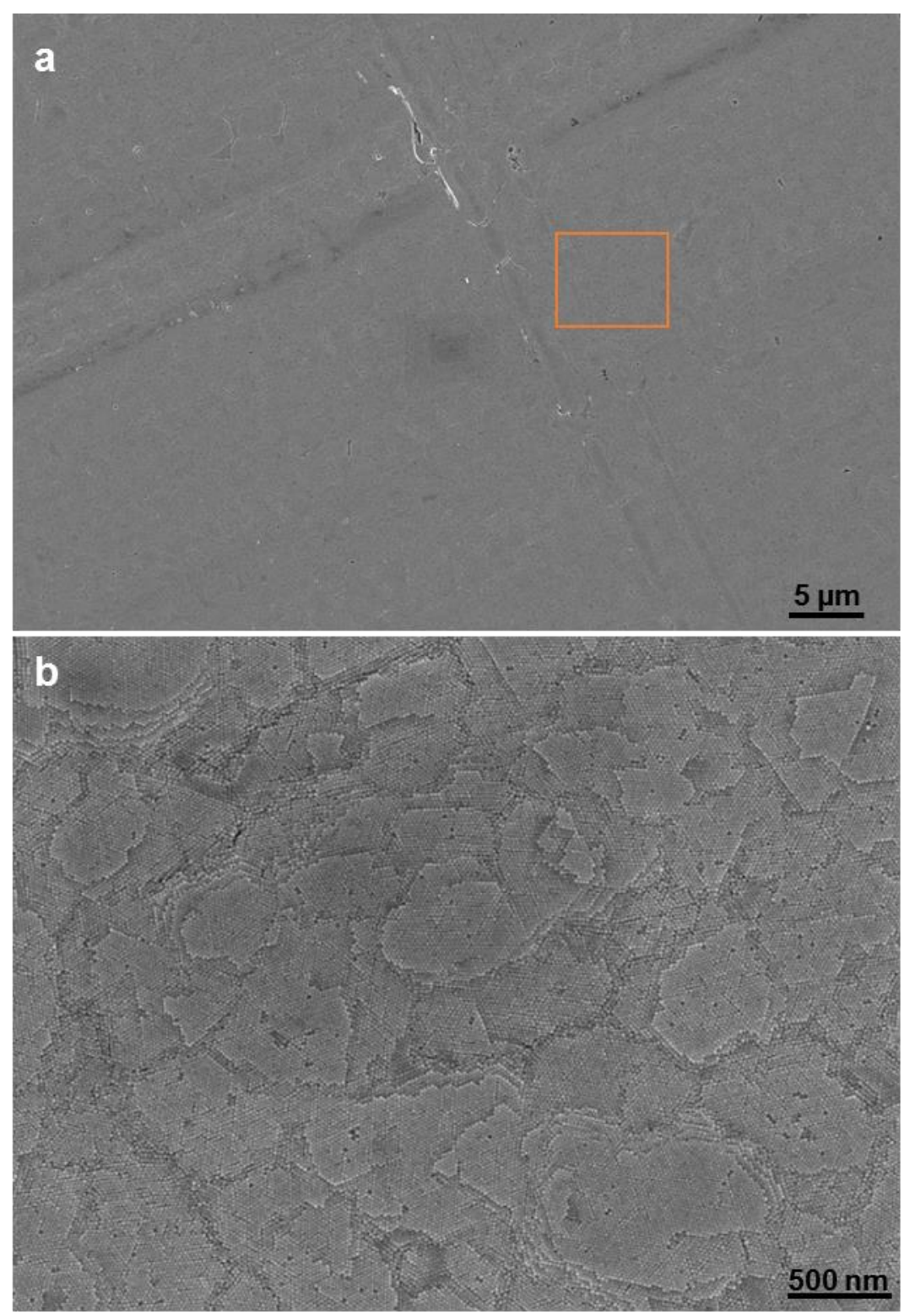

Figure S6. (a) Low-magnification SEM image of carbonized $\mathrm{Fe}_{3} \mathrm{O}_{4} \mathrm{NC}$ superlattice membranes grown by liquid-air interfacial assembly, showing the crack-free feature of the membrane over large areas. (b) High-magnification SEM image of the region indicated in (a), showing that NC membranes were composed of NC superlattice domains on the order of several hundred nanometers to a few micrometers. 


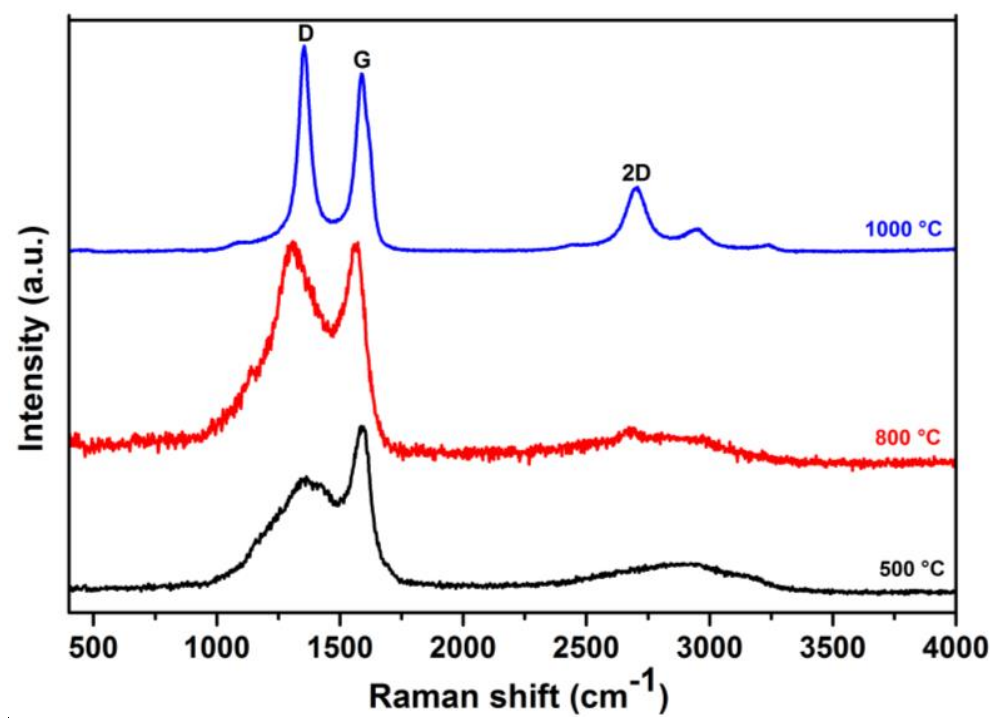

Figure S7. Raman spectra of MGF films heated at $500{ }^{\circ} \mathrm{C}$ and $800{ }^{\circ} \mathrm{C}$ under Ar for $2 \mathrm{~h}$. Raman spectrum of MGF films heated at $1000{ }^{\circ} \mathrm{C}$ under Ar for $2 \mathrm{~h}$ was also included for comparison. 

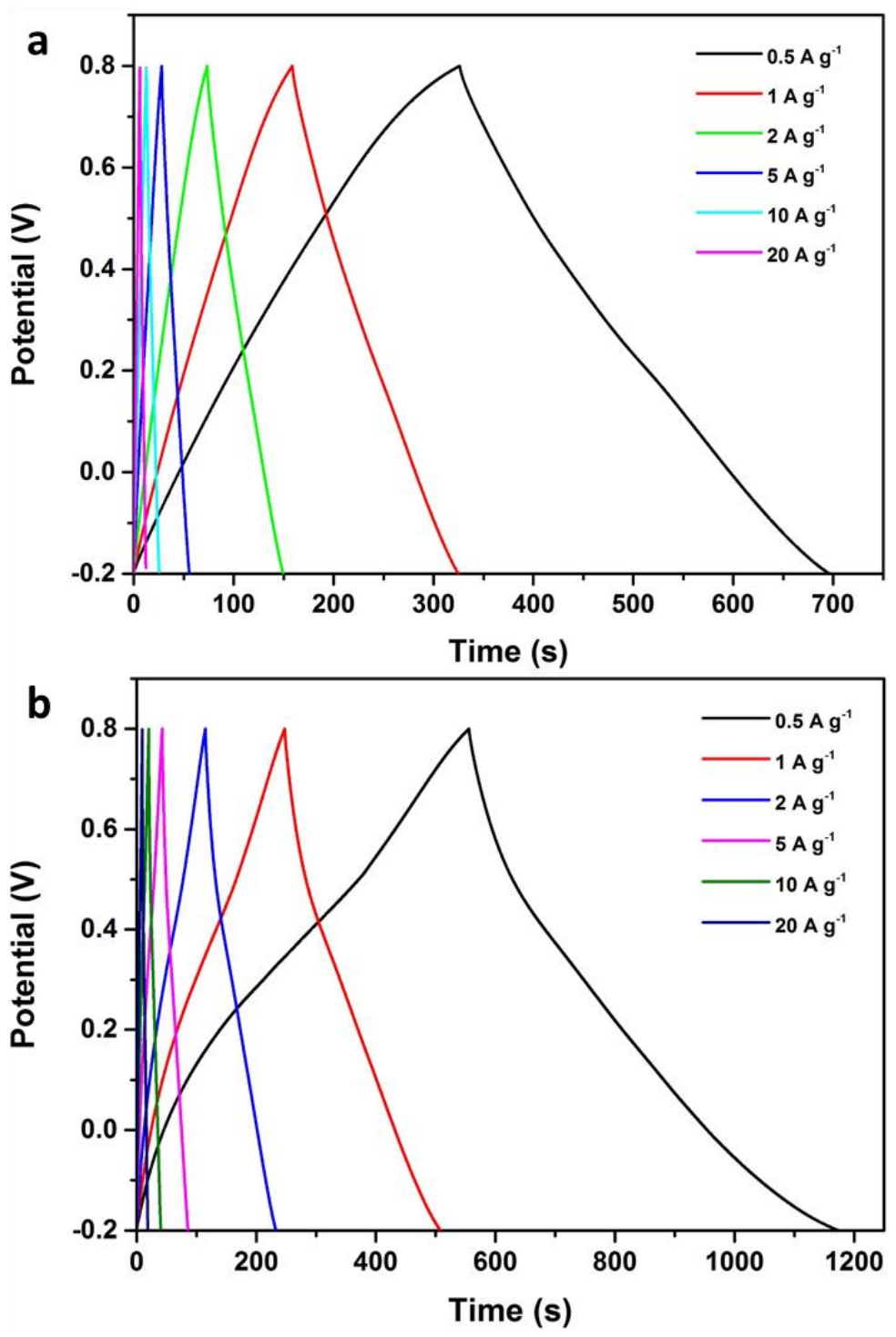

Figure S8. (a) Galvanostatic charge/discharge curves of MGF films measured under different current densities in $1 \mathrm{M} \mathrm{H}_{2} \mathrm{SO}_{4}$. (b) Galvanostatic charge/discharge curves of the acid-treated MGF films measured under different current densities in $1 \mathrm{M} \mathrm{H}_{2} \mathrm{SO}_{4}$. 


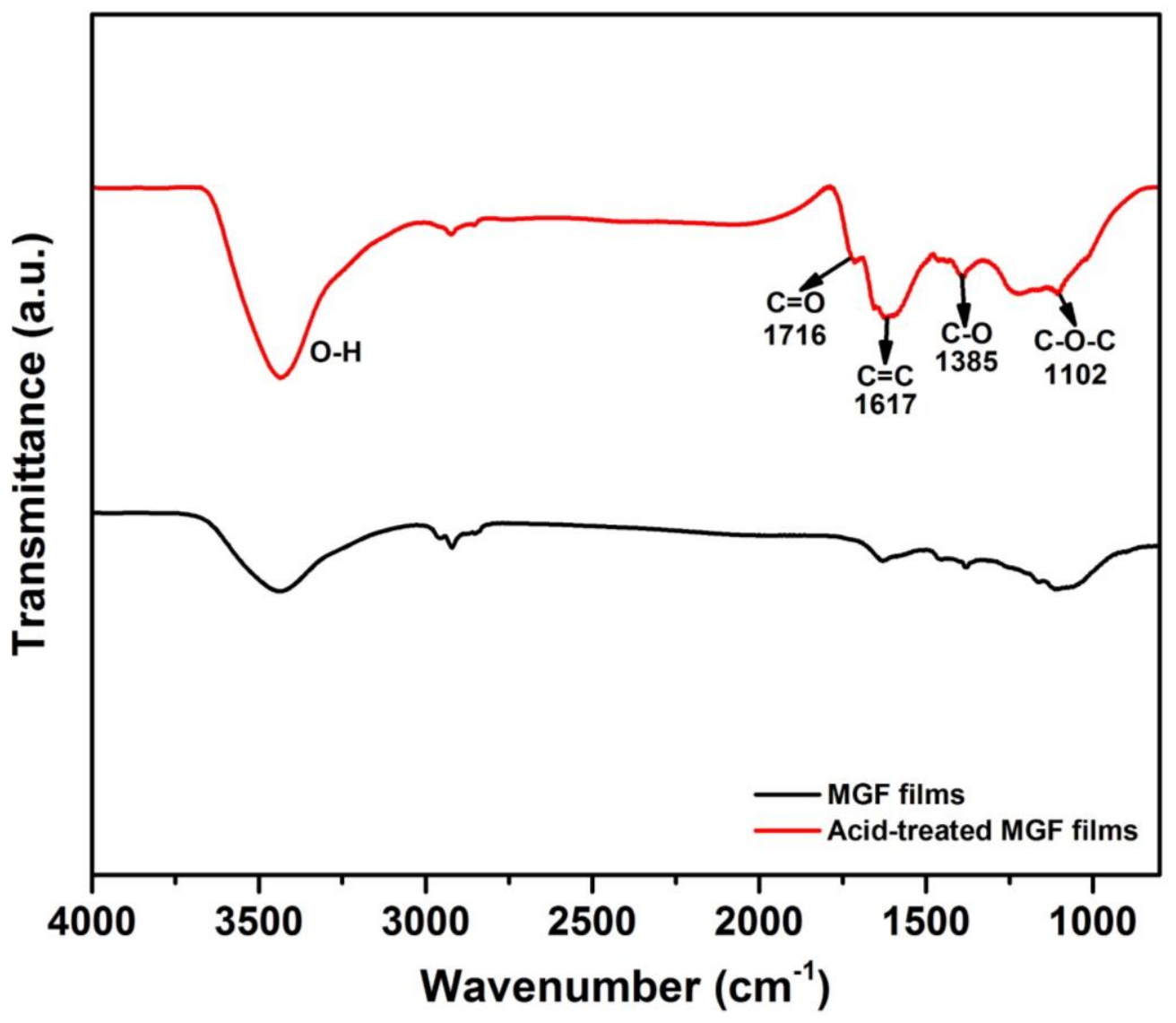

Figure S9. FTIR spectra of MGF films and the acid-treated MGF films. 


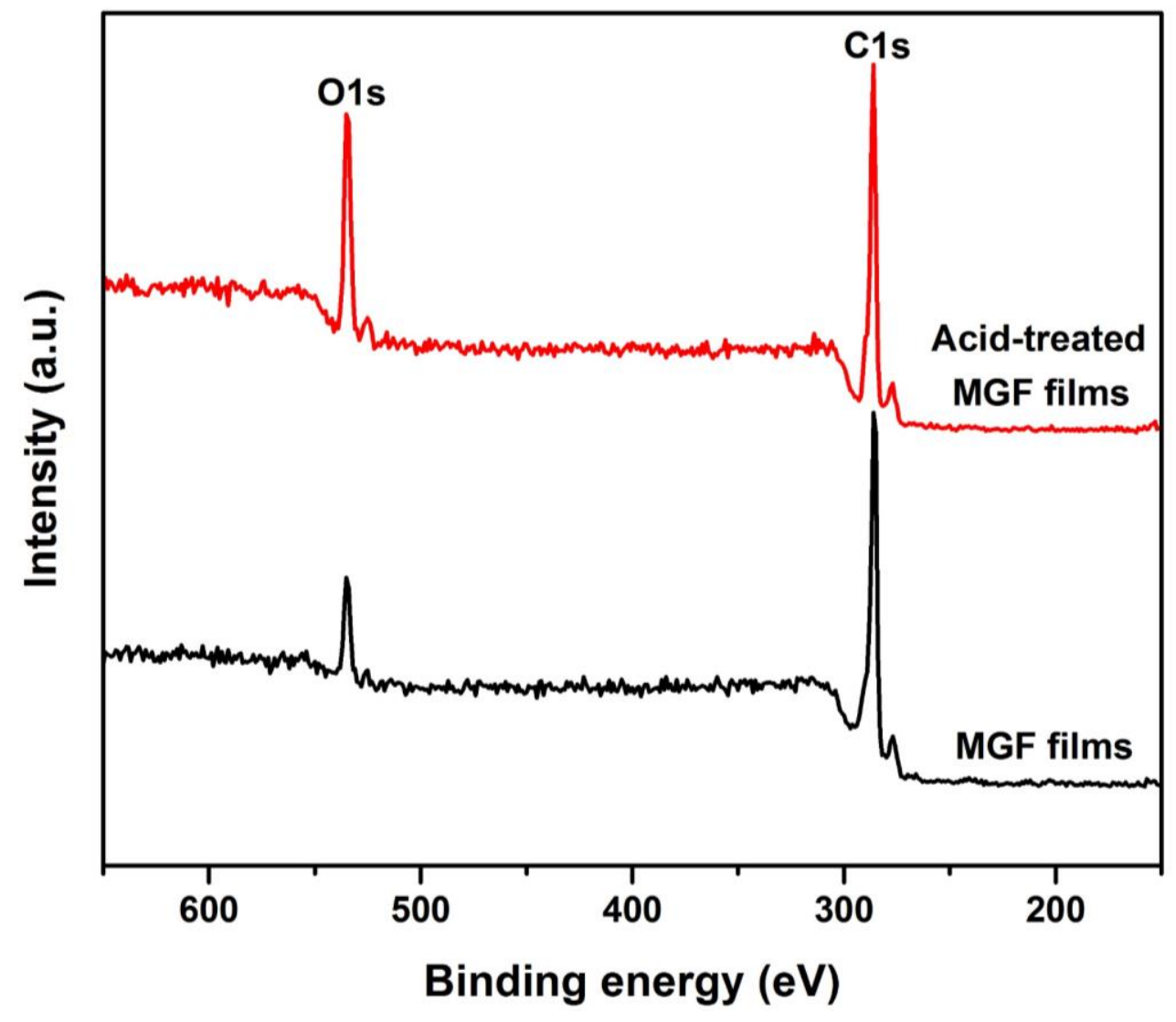

Figure S10. XPS spectra of MGF films and the acid-treated MGF films, respectively. 


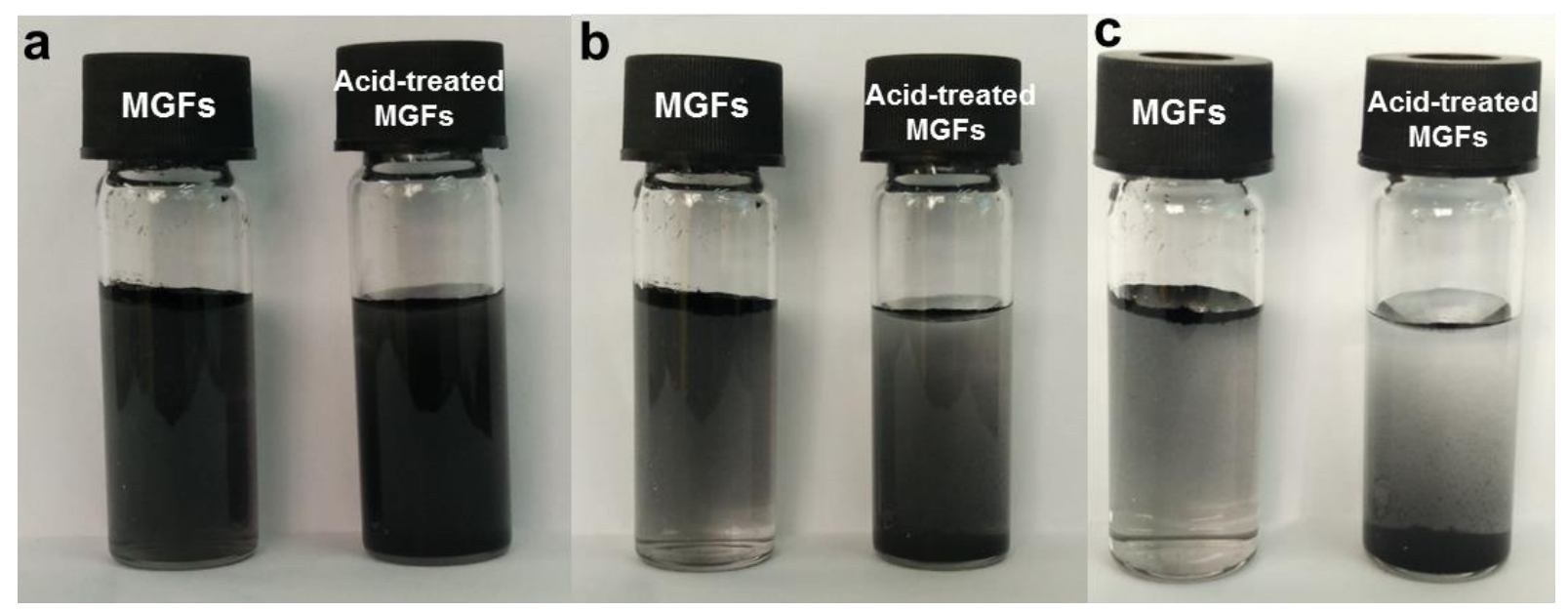

Figure S11. Dispersity test of MGFs and the acid-treated MGFs in water (a) just after, (b) at $1 \mathrm{~h}$ after, and (c) at $2 \mathrm{~h}$ after ultrasonication. Unlike the pristine MGF films which tended to float on the water surface, the acid-treated MGFs tended to stay in water before precipitation owing to the improved surface wetting properties. 


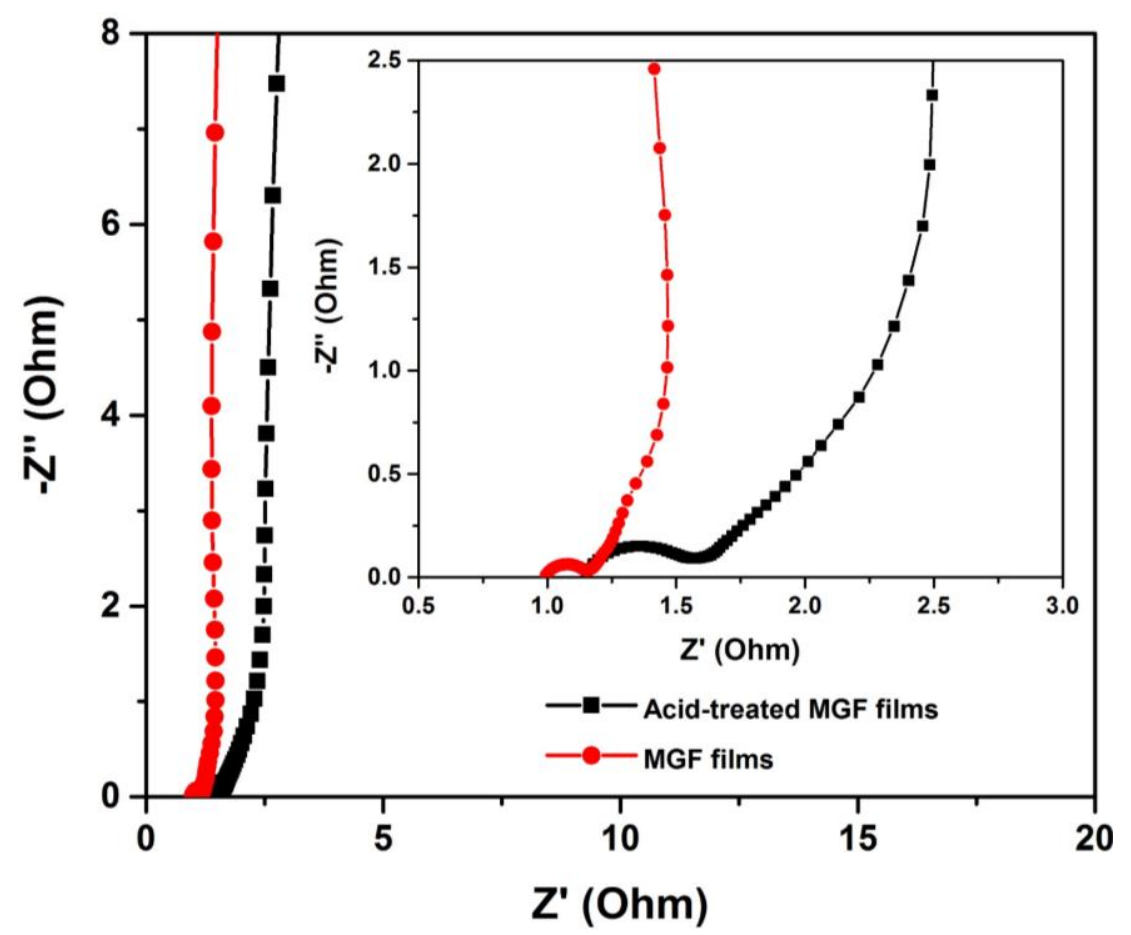

Figure S12. Nyquist plots of MGF films and the acid-treated MGF films in $1 \mathrm{M} \mathrm{H}_{2} \mathrm{SO}_{4}$. The inset shows an expanded view in high frequency and middle frequency region. 

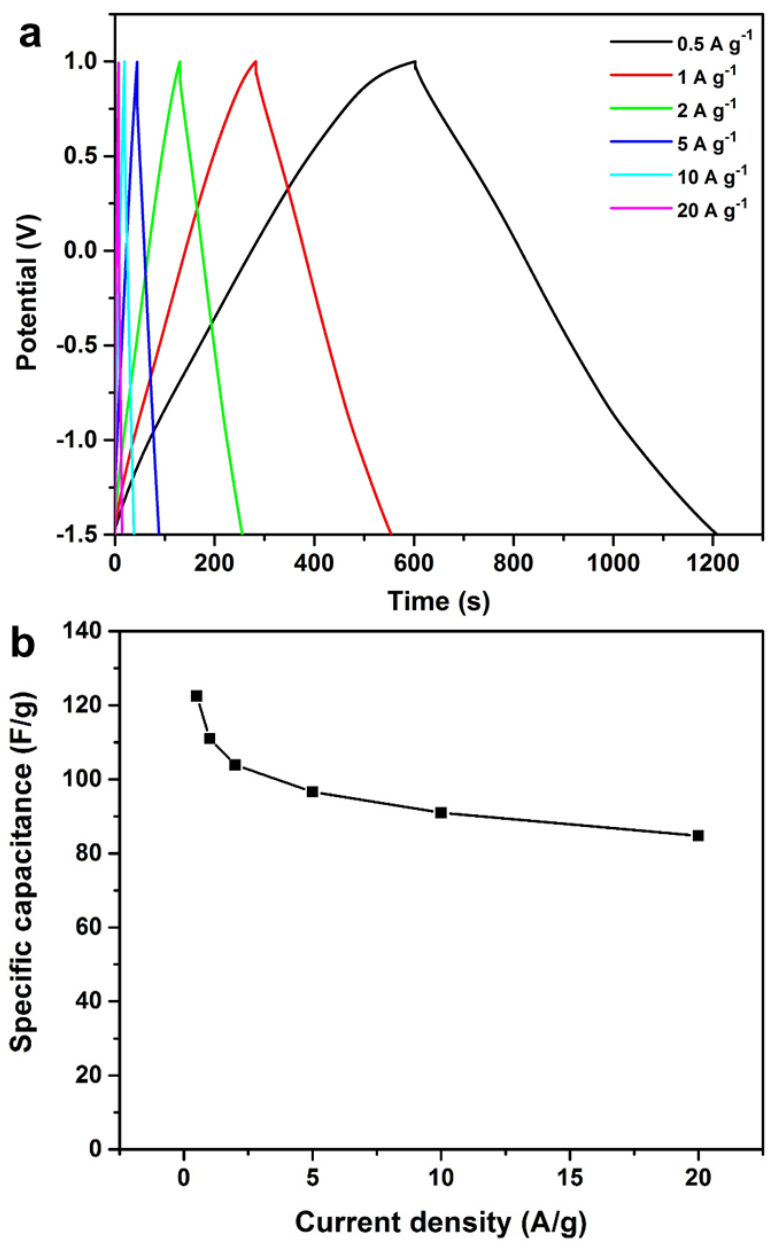

Figure S13. (a) Galvanostatic charge/discharge curves of MGF films measured under different current densities in $1 \mathrm{M} \mathrm{TEABF}_{4} / \mathrm{PC}$. (b) Specific capacitance as a function of current densities for MGF films measured in $1 \mathrm{M} \mathrm{TEABF}_{4} / \mathrm{PC}$. 

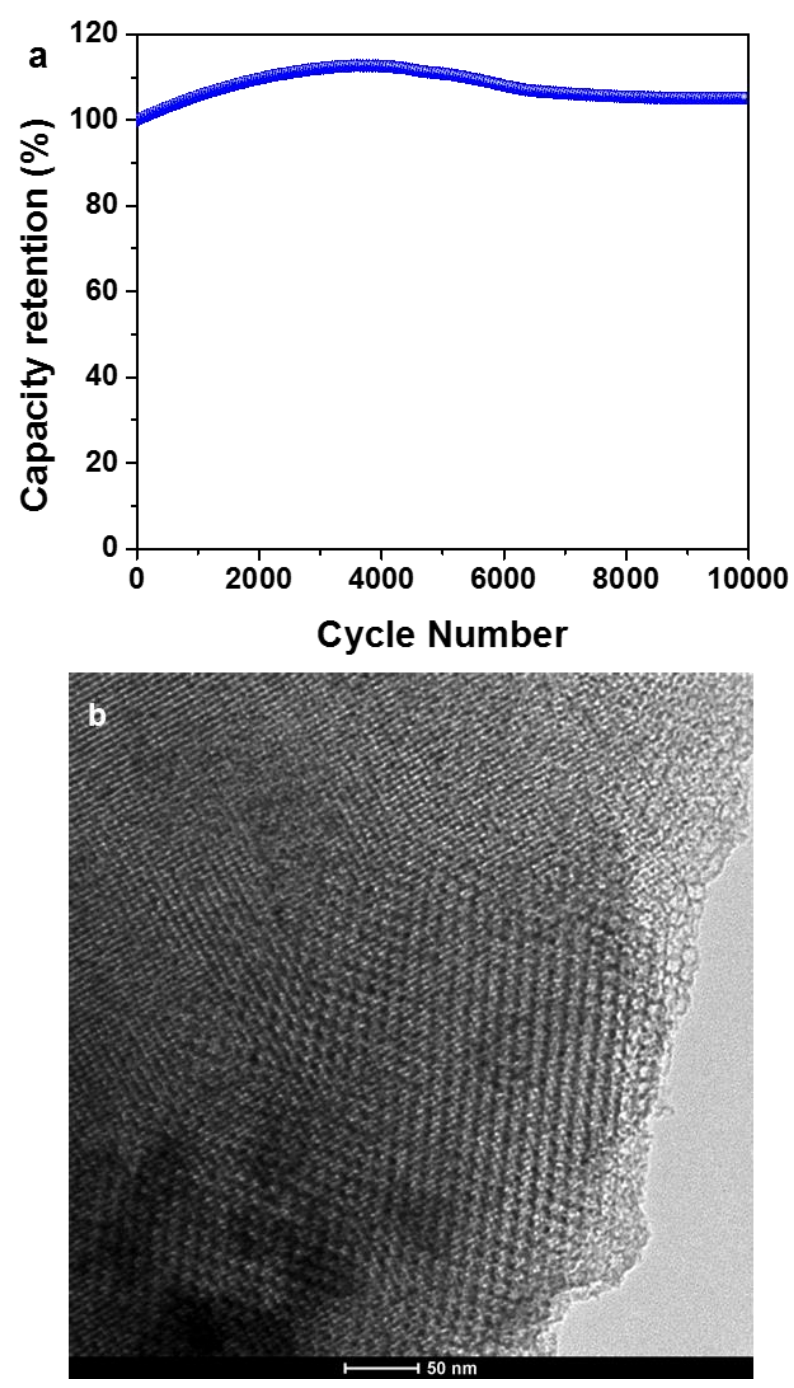

Figure S14. (a) Cycling stability of MGF films measured in $1 \mathrm{M} \mathrm{TEABF}_{4} / \mathrm{PC}$ at a current density of $20 \mathrm{~A} / \mathrm{g}$. (b) TEM image of MGF films after 10000 cycles, showing the well-retained ordered mesoporosity. 

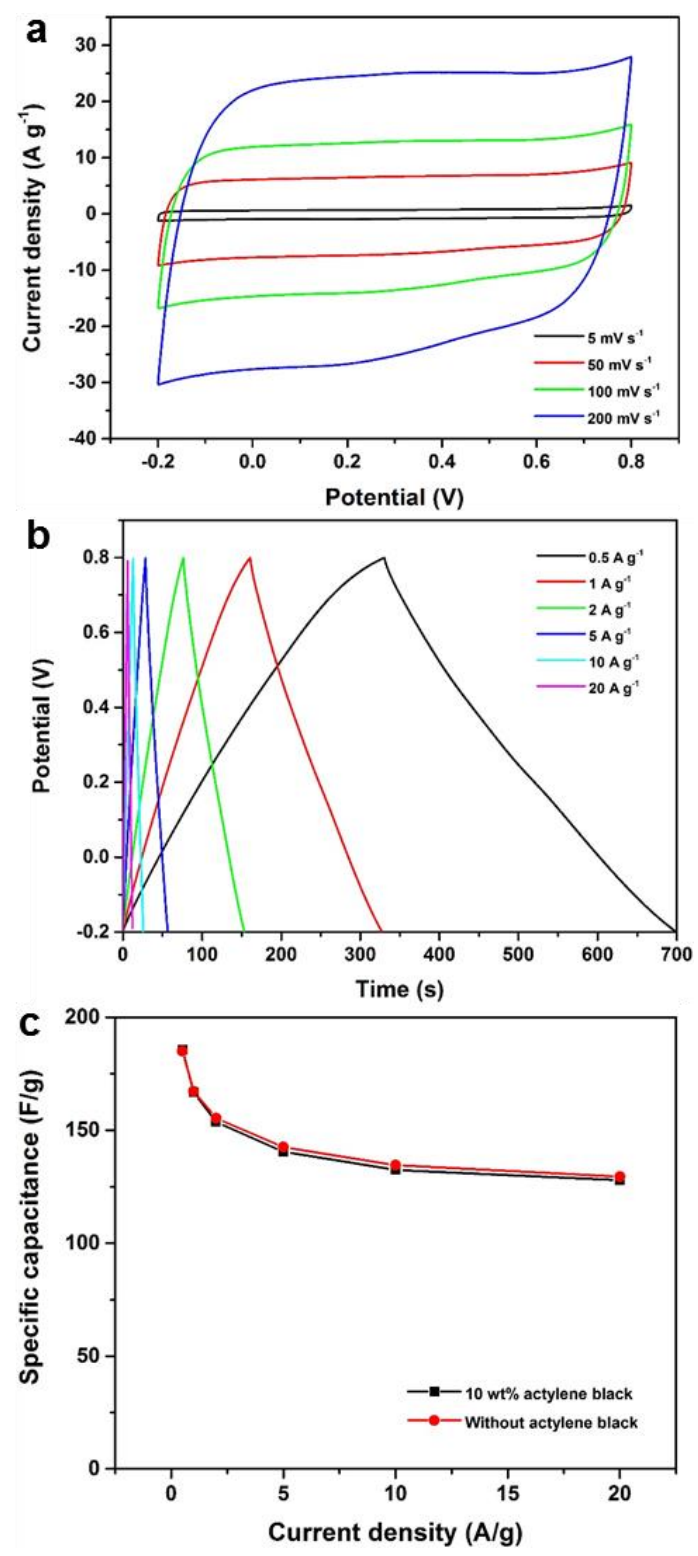

Figure S15. Electrochemical characterization of MGF films without the additional acetylene black in $1 \mathrm{M} \mathrm{H}_{2} \mathrm{SO}_{4}$. (a) $\mathrm{CV}$ curves. (b) Galvanostatic charge/discharge curves under different current densities. (c) Specific capacitance as a function of current densities. The capacitance change of MGF films with the additional acetylene black (10 wt $\%$ ) was also included for comparison. 

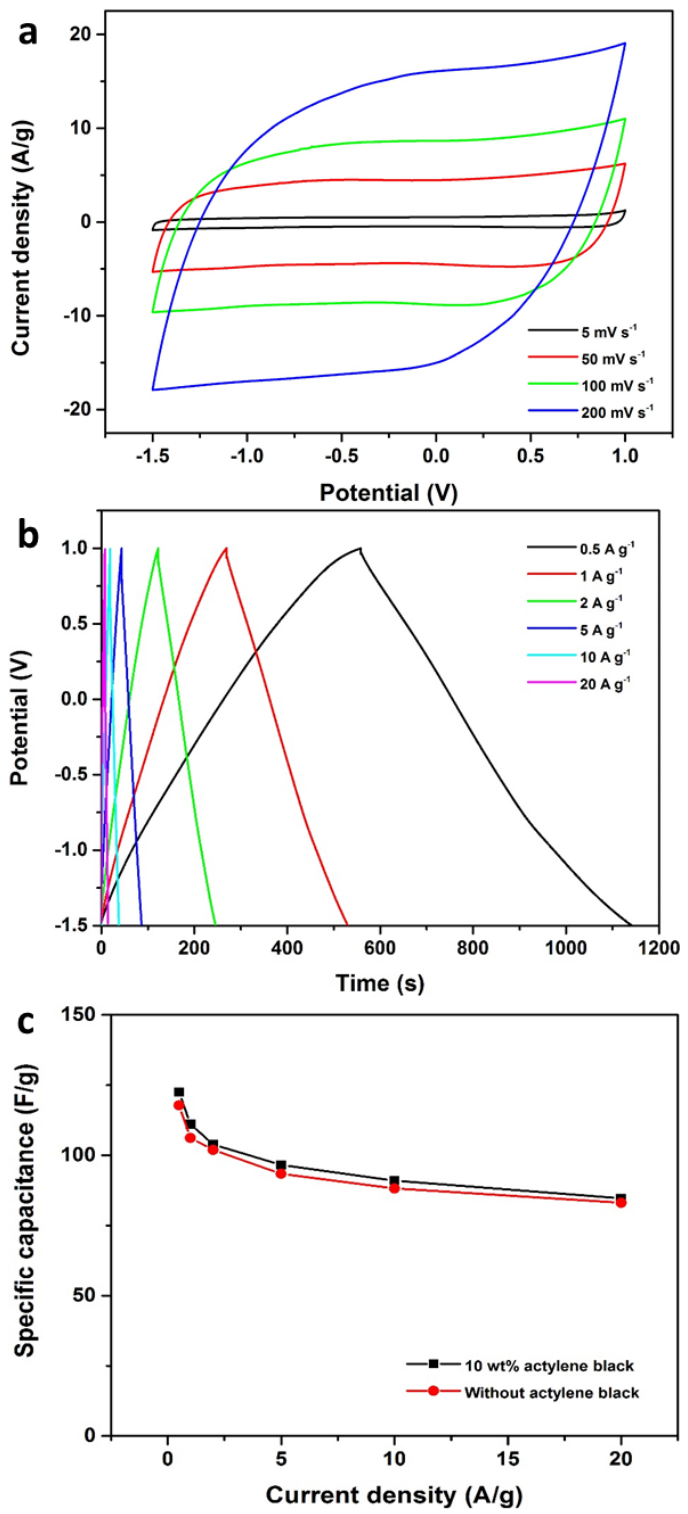

Figure S16. Electrochemical characterization of MGF films without the additional acetylene black in $1 \mathrm{M} \mathrm{TEABF} 4 / \mathrm{PC}$. (a) $\mathrm{CV}$ curves. (b) Galvanostatic charge/discharge curves under different current densities. (c) Specific capacitance as a function of current densities. The capacitance change of MGF films with the additional acetylene black (10 wt $\%$ ) was also included for comparison. 

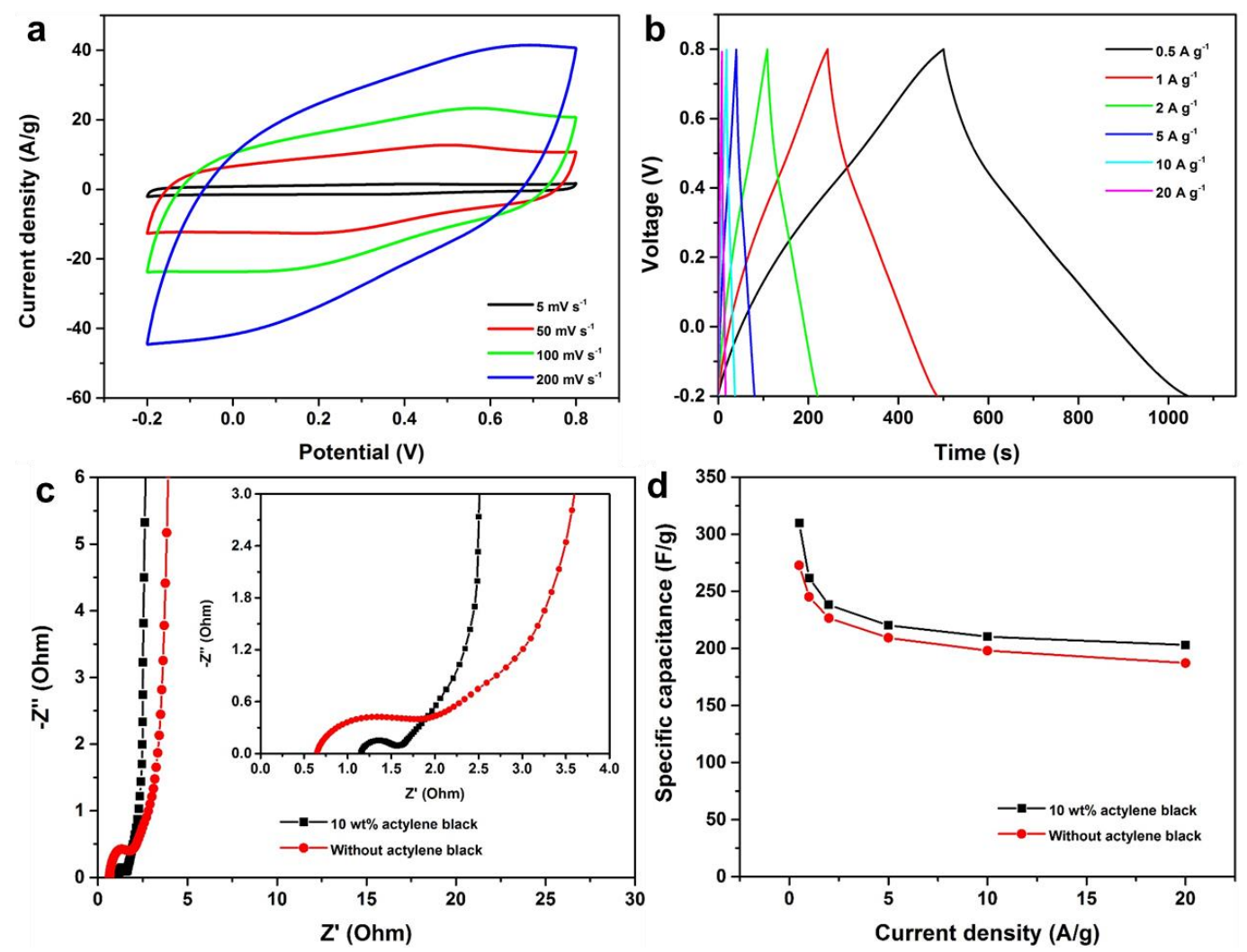

Figure S17. Electrochemical characterization of the acid-treated MGF films without the additional acetylene black in $1 \mathrm{M} \mathrm{H}_{2} \mathrm{SO}_{4}$. (a) $\mathrm{CV}$ curves. (b) Galvanostatic charge/discharge curves under different current densities. (c) Nyquist plot. The inset shows an expanded view in high frequency region. Nyquist plot of the acid-treated MGF films with the additional acetylene black (10 wt\%) was also included for comparison. (d) Specific capacitance as a function of current densities. The capacitance change of the acid-treated MGF films with the additional acetylene black (10 wt \%) was also included for comparison. 


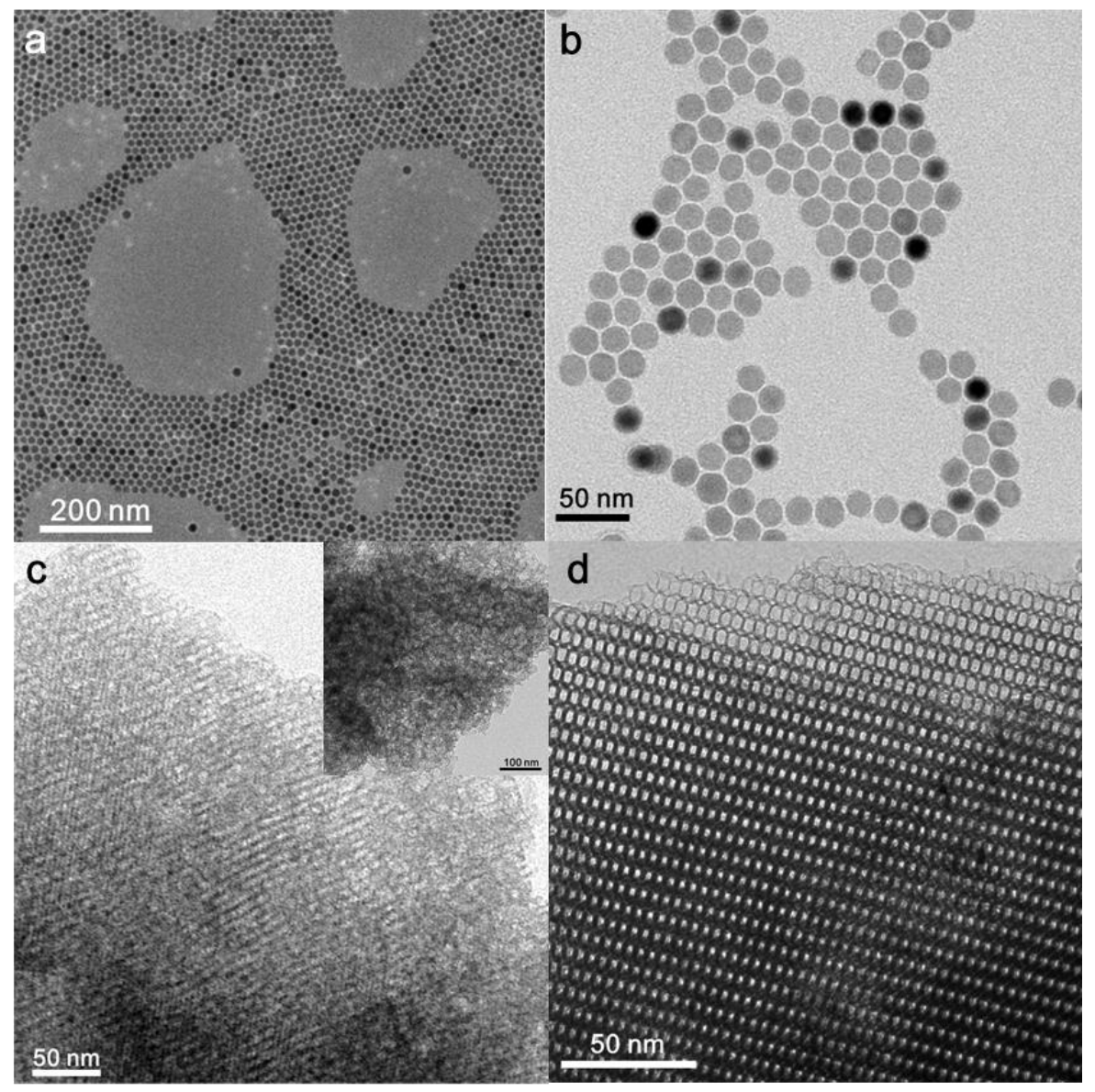

Figure S18. (a) TEM image of $8 \mathrm{~nm} \mathrm{CoFe} 2 \mathrm{O}_{4}$ NCs. (b) TEM image of $18 \mathrm{~nm} \mathrm{Fe} \mathrm{O}_{4} \mathrm{NCs}$. (c) TEM image of the ordered domain of MGF films derived from $8 \mathrm{~nm} \mathrm{CoFe}_{2} \mathrm{O}_{4} \mathrm{NCs}$. The inset shows the disordered or collapsed domain of the same MGF films. (d) TEM image of MGF films derived from $18 \mathrm{~nm} \mathrm{Fe}_{3} \mathrm{O}_{4} \mathrm{NCs}$. 

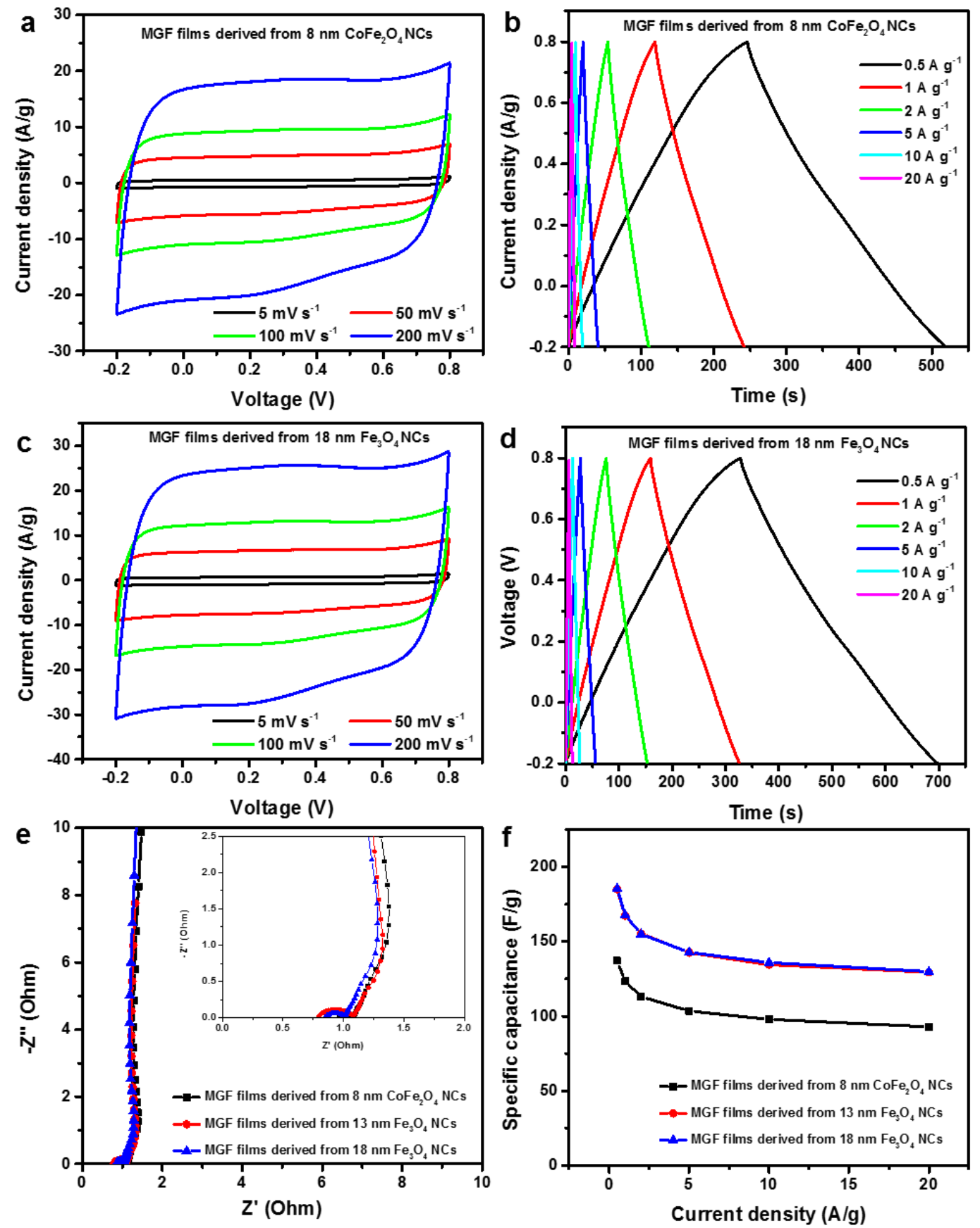

Figure S19. (a) CV curves at different scan rates and (b) Galvanostatic charge/discharge curves under different current densities of MGF films derived from $8 \mathrm{~nm} \mathrm{CoFe} 2 \mathrm{O}_{4} \mathrm{NCs}$ in $1 \mathrm{M} \mathrm{H}_{2} \mathrm{SO}_{4}$. (c) $\mathrm{CV}$ curves at different scan rates and (d) Galvanostatic charge/discharge curves under different current densities of MGF films derived from $18 \mathrm{~nm} \mathrm{Fe} 3 \mathrm{O}_{4} \mathrm{NCs}$ in $1 \mathrm{M} \mathrm{H}_{2} \mathrm{SO}_{4}$. (e) Nyquist plots of MGF films with different mesopore sizes in $1 \mathrm{M} \mathrm{H}_{2} \mathrm{SO}_{4}$. The inset shows an expanded view in high frequency region. (f) Specific capacitance as a function of current densities for MGF films with different mesopore sizes in $1 \mathrm{M} \mathrm{H}_{2} \mathrm{SO}_{4}$. 